\title{
Trade Networks and the Strength of Strong Ties*
}

\author{
Áureo de Paula ${ }^{\dagger}$
}

April 10, 2020

\begin{abstract}
Evidence suggests that, in the presence of imperfect market institutions, individuals devote resources to the establishment of reliable connections in order to attenuate the frictions that reduce trading and insurance opportunities. In this paper I survey the relevant literature on strategic formation of networks and use it to study this particular economic situation. A simple model is built to show that the investment in strong ties often, though not always, produces stable configurations that manage to improve upon the imperfections of market institutions. JEL Classification Numbers: C70, D20.
\end{abstract}

${ }^{*}$ The first version of this article was written in 2002 while I was in graduate school at Princeton University and first learned about the network formation literature. I thank José Scheinkman for stimulating my curiosity on the topic and Christian Julliard, Gabor Virag and Wei Xiong for invaluable comments on an earlier version of the paper.

${ }^{\dagger}$ University College London, CeMMAP and IFS, a.paula@ucl.ac.uk 


\section{Introduction}

The American Heritage Dictionary defines a network as (among other things) "an extended group of people with similar interests or concerns who interact and remain in informal contact for mutual assistance or support". Families and gangs are typical networks, but many other social and economic groups and situations can be framed similarly. As a matter of fact, sociology and other social sciences have explored this idea in many different contexts, from the "small world phenomenon" to needle sharing among drug addicts (for an introduction to the social networks literature, see Wasserman and Faust [30]). Networks also play a significant role in the economic arena. For instance, interpersonal contacts can be instrumental in job acquisition (e.g. Granovetter [16], Boorman [5] and Topa [29]), in the trade and exchange of goods (e.g. Kranton and Minehart [23] and Corominas-Bosch [9]), in providing insurance in underdeveloped communities (e.g. Fafchamps and Lund [13]) and in the diffusion of innovations (e.g. Rogers and Shoemaker [26]). Other examples could be cited, but these suffice to advocate the importance of better understanding the formation and consequences of social networks for economic behavior. This research agenda can actually be seen as part of the interest among economists in the feedbacks between social interactions (not mediated through markets) and economic institutions and behavior (e.g. Akerlof [1] and Postlewaite [25]).

In fact, group formation theories are not new in economics: from a strategic viewpoint, for instance, coalitional games have been around for about seventy years ${ }^{1}$. The more recent strategic network formation theories are naturally related to this literature, but allow for an even richer environment. In these models not only the composition of a coalition matters, but also the internal organization of each group: a given coalition may achieve very different results according to how its members relate to each other. Within this framework one can consider nuances such as the existence of costly ties and group benefits that flow through direct as well as indirect acquaintances.

\footnotetext{
${ }^{1}$ Examples of non-strategic approaches to problems of group formation are Tiebout [28] and Buchanan [6]
} 
Interesting phenomena arise under these circumstances, such as the tension between efficiency and stability of a social network that is present in many of these studies.

Whereas the mere establishment of links explored in the strategic models cited above already sparks several of facts and questions, extensions in some directions arise naturally. In a celebrated paper, for instance, Granovetter [15] suggests that as interpersonal links are allowed to vary in strength curious interactions come to pass. The author interprets the strength of a link as intuitively related to measures of emotional intensity, amount of time spent as well as the benefits and services provided by the tie. One of the main insights of the paper is that strong ties tend to cluster ${ }^{2}$, causing the diffusion of information and/or influence within the network of strong interpersonal links to be short-lived as only a reduced number of individuals - those within the strong links cluster where the information originates - will be reached. As a consequence, weak ties gain relevance as bridges between the different cliques of the population, working as the main highway for the diffusion of benefits. The author then goes on to support the "strength of weak ties" with a variety of informal evidences as well as a more careful investigation on the importance of personal contacts for job acquisition. One of my goals is to incorporate the concept of tie intensity in a strategic network formation model.

In his paper, Granovetter suggests that, under some circumstances, "formal" or mass procedures for diffusion of information or benefits can appropriately be treated as limiting cases of weak linkage chains. I follow this insight in representing markets as economic institutions in which individuals are brought together through a web of "weak ties". Under such representation, the connections operate to guarantee the transmission of trade opportunities within a certain community.

Some casual observation nevertheless shows that the fabric supporting this particular economic institution is neither uniform nor frictionless. Transaction costs of various sorts, search frictions and many other imper-

\footnotetext{
${ }^{2}$ If $A$ and $B$ are each strongly connected to $C$, it is very likely that $A$ and $B$ be strongly linked.
} 
fections are usually seen in economic life and may significantly reduce the dissemination of trade opportunities among a certain set of agents . When the weak linkages that put together market institutions are subject to noise, it is natural to expect the emergence of "voluntary networking": individuals will spend resouces (time, effort, money) establishing strong contacts that help attenuate the information and flow frictions which hinder the full realization of trade opportunities. In developing societies, for instance, where formal markets are subject to significant noise, informal networks for trade or insurance purposes are not rare events ${ }^{3}$. The formation of informal networks can be seen as well in small specialized markets such as markets for collectibles or rare books in which "connections" are very important. These markets can also be thought to be subject to significant friction if one is to rely solely on formal or casual trade opportunities.

When should we expect to see the apperance of such interpersonal networks as a stable support of economic transactions? Having been established as a stable phenomenon, does voluntary networking improve upon the situation in which no such connections can be established? In this paper I extend a trade network first suggested by an example in Jackson and Watts [21] to investigate these questions.

The rest of the paper is organized as follows: Section 2 presents a survey of the relevant literature on strategic network formation, the following section sets up a very simple framework to investigate the properties of a trade model that incorporates different intensities for interpersonal links. Section 4 concludes.

\section{Review of the Literature}

This section presents a brief survey of the recent work on strategic formation of networks ${ }^{4}$. Before this is done though, I present a generic description of

\footnotetext{
${ }^{3}$ See Fafchamps and Lund [13] for an empirical investigation on informal insurance networks in rural Philippines and Genicot and Ray [14] for a theoretical investigation using a framework closer to the traditional coalitional game literature

${ }^{4}$ The section focuses particularly on issues of stability and efficiency. For broader views of this literature, see Dutta and Jackson [11] and Jackson [19].
} 
the object investigated in the paper: a strategic model of network formation.

\section{$2.1 \quad$ A Network Formation Game}

A graph is a collection of binary subsets of a given finite set, whose elements are called the vertices of the graph. Each of these binary subsets corresponds to an edge or a link between a pair of vertices. Two different elements can be connected directly or indirectly through a series of intermediaries, which determine a path between two elements. These objects are easily represented graphically. For instance, taking as primitive a set with $n=8$ elements represented by dots, each the following figures relates to a different graph. Figure 1A represents an empty graph, in which there are no edges. Figure 1B depicts a complete graph, in which all the vertices are linked. Figure 1C shows a graph with two components (isolated subgraphs).

\section{FIGURE 1 HERE}

Graphs are natural representations for networks in many different contexts. They are also a basic ingredient of a strategic model of network formation. Such a game can be generically described by the tuple $\Gamma=$ $\left\langle N,\left(A_{i}\right)_{i \in N}, v, Y\right\rangle$, consisting of:

- A finite set $N=\{1, \ldots, n\}$ (the set of players);

- For each player $i$ a set $A_{i}$ (the set of possible strategies) where $A_{i}=\{$ all binary subsets of $N$, ordered or not, depending on the context, containing $i\}$ or $A_{i}=\{$ all ordered pairs $(i, j): j \in N \backslash\{i\}\}$;

- A function $v:\left\{G \mid G \subset G^{N}\right\} \rightarrow \Re$ (the value of a graph) where $G^{N}$ is the set of all networks on $N$; and

- A function $Y:\left\{G \mid G \subset G^{N}\right\} \times v \rightarrow \Re^{N}$ (the allocation rule).

It is also common practice to represent a strategy by a vector $g_{i}=$ $\left(g_{i 1}, \ldots, g_{i i-1}, g_{i i+1}, \ldots, g_{i n}\right)$ where $i \in N$ and $g_{i j} \in\{0,1\}$ for each $j \in N \backslash\{i\}$. When agent $i$ 's strategy includes the formation of a link with another agent 
$j$ (i.e. $\left.\{i, j\} \in A_{i}\right) g_{i j}=1$, while $g_{i j}=0$ otherwise. We use the notation $g_{T}$ to denote a strategy profile $\left(g_{i}\right)_{i \in T}$ chosen by the players in the set $T \subseteq N$. Agents are assumed to prefer higher payoffs. The payoff structure is captured by the allocation rule. This allocation structure may represent many things. When a pure social network is considered and utility is not transferable, the allocation rule stands for the utility ascribed to each individual. When the network relates to a production or trade architecture, the allocation rule may correspond to the share of each player, the outcome of a bargaining process or some other form of redistribution. In some applications the value of a graph is simply the sum of each agent's utility under a given configuration $\left(v(G)=\sum_{i=1}^{n} u_{i}(G)\right.$, where $\left.u_{i}:\left\{G \mid G \subset G^{N}\right\} \rightarrow \Re\right)$. As mentioned in the introduction, the flexibility of the above model allows for the consideration of costly ties and indirect benefits from individuals not directly connected to a player, but accessible through a path of adjacent neighbors.

The strategy space $A_{i}$ is represented by sets of binary sets or ordered pairs according to whether a directed or non-directed graph is the suitable network model. Diffusion is unidirectional on each arc in a directed graph, which is often represented diagrammatically by arrows instead of segments linking two vertices. These structures are usually applied to situations in which the costs of a tie are asymmetric, when the establishment of the link $g_{i j}=1$ imply costs to $i$ but not to $j$ (clicking on $j$ 's website or reading a paper written by $j$, for instance).

Once each agent picks a strategy, a graph is formed as the outcome of the game: the network structure that will allow the diffusion of benefits through the links established between the players. The papers in the literature have come up with a series of solution concepts in order to assess the stability of a given structure. These concepts vary according to the way in which the game is set up and might relate to categories in standard non-cooperative theory, in the cooperative game theory tradition or some sort of compromise between both. Some of the equilibrium notions used are pairwise stability (Jackson and Wolinsky [21]), Nash networks (Bala and Goyal [2]) and weak and strong stability (Dutta and Mutuswami [12]). A detailed presentation 
of these concepts is left for a later subsection.

Another concept that raises attention in the literature is that of strong efficiency of a given network. This notion is distinct from that of Pareto efficiency and, as highlighted by Jackson [18], is more suitable when value is fully transferable and intervention is possible. Pareto efficiency, on the other hand, would be more appropriate when the allocation rule is fixed and payoffs are not tranferable ${ }^{5}$.

Definition 1 (Strong Efficiency) A network $G \subset G^{N}$ is strongly efficient if $v(G) \geq v\left(G^{\prime}\right)$ for all $G^{\prime} \subset G^{N}$.

A tension between stability and efficiency is very common in many of the works.

\section{$2.2 \quad$ Examples}

Below I present a sample of specific models. These examples are often discussed in the literature as a device to motivate the analysis in more abstract settings.

1. The Connections Model (Jackson and Wolinsky [21]) Players establish ties in order to communicate. The benefit of the information obtained by a player $i$ from a vertex $j$ has value equal to 1 , whereas the cost of maintaining a direct link with this individual is given by $c>0$. The connection cost $c$ is incurred by both vertices of a link. Agents can benefit from indirect contact with other players, but costs are incurred only on direct contacts. The utility of each node on a graph $g$ is then assumed to be:

$$
u_{i}(G)=\sum_{i \neq j} \delta^{t_{i j}}-\sum_{j:\{i, j\} \in G} c
$$

where $0<\delta<1$ suggests the idea that there is some decay in the transmission of value - closer individuals are more valuable - and $t_{i j}$ is

\footnotetext{
${ }^{5}$ I make this distinction following the characterization given by Jackson [18], although this same author stresses elsewhere (Jackson and Wolinsky [21]) that the two concepts (strong and Pareto efficiency) coincide when utility is transferable.
} 
the number of links in the shortest path between $i$ and $j$ (also known as geodesic distance and assumed to be $\infty$ when no path exists between $i$ and $j$ ).

For this model $v(G)=\sum_{i \in N} u_{i}(G)$ and $Y_{i}(v, G)=u_{i}(G)$. In establishing connections with other players an agent will take into consideration the costs and benefits of direct ties as well as the indirect benefits that a link might provide.

2. The Co-Author Model (Jackson and Wolinsky [21]) In this model, each agent is a researcher that has to decide whether to establish co-authorship links with other researchers. The production of an paper consumes time and the more committed the co-author is to other projects, the lower his or her potential contribution is to someone considering him or her for a new project. The utility of an individual that is involved in $n_{i}$ projects on a graph $G$ is postulated to be:

$$
u_{i}(G)=\sum_{j:\{i, j\} \in G} \frac{1}{n_{i}}+\frac{1}{n_{j}}+\frac{1}{n_{i} n_{j}}
$$

when $n_{i}>0$, whereas for $n_{i}=0$ it is postulated that $u_{i}=0$. The output of each project is interpreted to be positively related to the time invested by each member in the project $\left(1 / n_{i}+1 / n_{j}\right)$ and on some measure of interaction captured by $1 / n_{i} n_{j}$ ). In contrast to the previous situation, here indirect links are harmful since they take up the co-author's attention. $v$ and $Y$ are given by the same expressions of $u_{i}$ as in the previous example.

3. The Trade Example (Jackson and Watts [20]): The players benefit from trading with other agents as long as they are linked (directly or not). Trade is assumed to flow without any decay or friction along any path, delivering a Walrasian equilibrium at each connected subgraph. The players begin by forming a network, then randomly receive endowments and trade with whoever belongs to his or her network component. This model is further specialized by Jackson and Watts to deliver an interesting situation in which no stable network exists 
(according to the concept of pairwise stability). This is the model I extend in section 3 in order to explore weak and strong connections in exchange situations.

\subsection{The Literature}

This subsection surveys a few of the papers in the literature, dedicating special attention to the issue of compatibility between efficiency and stability in the models. A more comprehensive view of the field can be found in Dutta and Jackson [11] or Jackson [19]. Most of the models can be understood through the framework of a network formation game developed earlier in this section.

One of the pioneering papers in the literature is Myerson [24]. In this paper, the author incorporates a network structure into a traditional transferable payoff coalitional game represented by $\langle N, v\rangle$, meant to capture possible communication and/or cooperation channels among the players of the game. A graph would list connections between agents and restrict the possible formation of coalitions to those among individuals belonging to a same connected subgraph. The object generated in this setting, comprising the original coalitional game $\langle N, v\rangle$ and the graph $G$, is called a graph-restricted game $\left\langle N, v^{G}\right\rangle$. Using this structure Myerson explores some extensions of traditional solution concepts in cooperative game theory, such as the Shapley value (which in this framework is usually called the Myerson value).

Though providing an invaluable extension of coalitional game theory, the structure developed by Myerson still exhibited a reduced degree of flexibility in the interaction between the cooperation architecture and payoff profiles. In a graph-restricted coliational game like the one introduced in Myerson [24], the network only restricts the possible coalitions to be formed and, as long as the players are connected, any graphical structure within a given coalition would deliver the same results. Jackson and Wolinsky [21] expanded the analysis of cooperation strutures by defining the value function directly on the network structure instead of the coalition composition. This introduces the possibility of costly links as well as indirect, decaying benefits from interpersonal links. Within this framework a given coalition 
can achieve very different results depending on the internal organization of the group. To investigate the relation between efficiency and stability of a social network, the authors introduce the notion of pairwise stability, which requires that no pair of agents be willing to establish a link that is not present and that no player be willing to sever existing ties with any of his or her partners. Formally:

Definition 2 (Pairwise Stability) The graph $G$ is pairwise stable with respect to $v$ and $Y$ if

(i) for all $\{i, j\} \in G, Y_{i}(G, v) \geq Y_{i}(G-i j, v)$ and $Y_{j}(G, v) \geq Y_{j}(G-i j, v)$ and

(ii) for all $\{i, j\} \notin G$, if $Y_{i}(G, v)<Y_{i}(G+i j, v)$ then $Y_{j}(G, v)>Y_{j}(G+i j, v)$ where $G+i j$ stands for $G \cup\{i, j\}$ and $G-i j$ means $G-\{i, j\}$. As remarked by the authors, this notion of stability is relatively weak and can be strengthened in many ways, but looks like a reasonable starting point for any notion of stability that one may devise. Jackson and Wolinsky initially analyze the properties of the connections and of the co-author models delineated earlier. In both models they find a tendency for stable networks to be over-connected from an efficiency perspective. In the connections model, this will happen for sufficiently high costs as no agent will wish to maintain links with another agent that does not bring additional new value from indirect ties. Though stable, this network could be made more productive as a whole - from the perspective of $v$-if individuals incurred in less connection costs. In the co-authors model, the tension arises as researchers disregard the negative externality they bring about on the productivity of his or her co-author with respect to other joint projects. In a more general setting, the authors find out that, for a natural class of value function $v$, no strongly efficient network is pairwise stable. Jackson and Wolinsky's results raise interesting questions as to the circumstances that guarantee the compatibility of stable and efficient networks.

In Jackson and Wolinsky [21] only homogeneous settings are considered. Johnson and Gilles [22] extend the connections model to allow for heterogeneous costs of link formation depending on some notion of distance between 
two individuals. Even though significantly different results from the original model are obtained concerning the archictecture of stable graphs, the tension between stable and efficient graphs remains.

Dutta and Mutuswami [12] further expand the analysis of this issue using a framework very similar to the one presented in Jackson and Wolinsky [21]. In the game, each player concomitantly announces the set of links he or she wants to form and whenever there is simultaneity of wants the tie is established. They work with the notion of strong and coaliton-proof Nash equilibria, instead of pairwise stability. These concepts are defined below.

Definition 3 (Strong Nash Equilibrium) The strategy profile $g^{*}=\left(g_{1}^{*}, \ldots, g_{n}^{*}\right)$ is a strong Nash equilibrium with respect to $v$ and $Y$ if there exists no $T \subseteq N$ and no strategy profile $g=\left(g_{1}, \ldots, g_{n}\right)$ such that

(i) $g_{i}=g_{i}^{*}$ for all $i \notin T$ and

(ii) $Y_{i}(G, v)>Y_{i}\left(G^{*}, v\right)$ for all $i \in T$ (where $G^{*}$ and $G$ are the graphs induced by $g_{N}^{*}$ and $\left.g_{N}\right)$.

This stability concept requires that the network be robust to deviations by groups of players. Notice that if a graph is the outcome of a strong Nash equilibrium, it necessarily is pairwise stable. The next concept requires that we define some new notation. For any $T \subset N$ and profile of strategies $g_{N \backslash T}^{*}=\left(g_{i}^{*}\right)_{i \in N \backslash T}$, we define the game induced on $T$ by $g_{N \backslash T}^{*}$ as

$$
\Gamma\left(v, Y, g_{N \backslash T}^{*}\right)=\left\langle T,\left(A_{i}\right)_{i \in T}, \bar{v}, \bar{Y}\right\rangle
$$

where $\bar{v}$ and $\bar{Y}$ are defined to be the functions $v$ and $Y$ restricted to graphs induced by strategy profiles in which the strategies of players in $N \backslash T$ are fixed at $g_{N \backslash T}^{*}$. The concept of coalition-proof Nash equilibrium is defined as follows ${ }^{6}$ :

Definition 4 (Coalition-proof Nash Equilibrium) In a single-player game, $g^{*}$ is a coalition-proof Nash equilibrium of the game $\Gamma$ if and only if $g_{i}^{*}$ maximizes $i$ 's payoff over the set of possible strategies available to $i$. If $\Gamma$ is an $n$ -

\footnotetext{
${ }^{6}$ See the original formulation in Bernheim, Peleg and Whinston [4].
} 
person game $(n>1)$ and assuming that a coalition-proof Nash equilibrium was defined for games with less than $n$ players, then (i) $g^{*}$ is self-enforcing if for all $T \subset N, g_{T}^{*}$ is a coalition-proof Nash equilibrium of $\Gamma\left(v, Y, g_{N \backslash T}^{*}\right)$; and (ii) $g^{*}$ is a coalition-proof of $\Gamma$ if it is self-enforcing and there does not exist another self-enforcing strategy vector $g$ such that provides a higher payoff to all players in the game.

Given these two equilibrium concepts, the following notions of stability are defined:

Definition 5 (Strong and Weak Stability) The graph $G^{*}$ is strongly stable (respectively weakly stable) with respect to $v, Y$ if $G^{*}$ is induced by a strategy profile that is a strong Nash equilibrium (respectively a coalitionproof Nash equilibrium).

Dutta and Mutuswami [12] study the very same problem analyzed by Jackson and Wolinsky [21], but from a somewhat different angle. Their approach consists of regarding the value function as a datum of the situation at hand, while the allocation rule shows some flexibility, possibly being "chosen" or "designed". They then take a "mechanism design" or "implementation" perspective: given a value function, can there be an allocation rule that delivers convenient efficiency and ethical properties for the equilibrium network? The authors find that such "mechanisms" are possible, though the nice properties required of the network do not necessarily hold out of the equilibrium. For situations in which the concept of stability is well understood and appropriate and intervention is possible, the results are then encouraging.

The models reviewed so far assume that the formation of a link involves costs for both parties involved. One consequence is that any stability concept has as a minimum requirement the notion of pairwise incentive compatibility. Bala and Goyal [2] consider instead situations in which connection costs are unilaterally incurred and a link prescinds from mutual consent (e.g., web links or one-sided compatibility of software). Networks generated under these circumstances are directed graphs, as opposed to the 
non-directed structures obtained under two-sided costs for the establishment of links. This structure allows for the analysis of stability notions that are even weaker than the ones defined previously, such as networks induced by Nash equilibrium strategy profiles. Bala and Goyal call these graphs Nash networks. They also analyze structures induced by strict Nash equilibria (strict Nash networks), where each agent gets a strictly higher payoff with his or her current strategy than he or she would with any other strategy. To study the stability-efficiency conflict, the authors take a different avenue. Instead of investigating this tension in a static setting, as done by the previous papers cited in this survey, learning dynamics is introduced. For this, a version of the best response dynamics is used, where the network game is played repeatedly and each individual chooses the set of links that maximizes his or her payoff given the graph of the previous period. For a few variations of the network formation model the results indicate that the network frequently converges to a stable and efficient architecture $^{7}$, which contrasts with the results obtained in a non-directed setting. Though encouraging, these results get a cautionary note in Dutta and Jackson [10], which indicate that the efficiency-stability tension may be significantly fragile in more general directed settings. Other interesting explorations on Nash networks can be cited. In an interesting extension, for instance, Heller and Sarangi [17] introduce agent heterogeneity and imperfect information to the model.

Although these papers comprise a representative sample of the recent literature on strategic network formation models, many unexplored possibilities remain. Dutta and Jackson [11] is a recommended reference for a broader view and other perspectives on the subject. One suggestion that seems to have received reduced attention in this literature, at least until the original writing of this note, is the sort of structure suggested by Granovetter [15]: the possibility of different intensities for the links established. Boorman [5] is one of the few papers to attack the idea at the time when

\footnotetext{
${ }^{7}$ Two graphs belong to the same architecture if one network can be obtained from the other by permuting the roles of the vertices.
} 
this note was first written ${ }^{8}$.

Boorman formally examines a symmetric labor market model like the one described in Granovetter's paper, where individuals choose to establish weak or strong ties with other players. Here, the transmission of job information follows a priority rule according to which a job offer not taken by the person that first learns about it is forwarded to the circle of friends with strong ties. In case they refuse it, the job news is sent to other acquaintances, those in the weak-tie neighborhood. The model presented in Boorman's paper is closely related to the analysis of Nash networks and this is the concept of stability adopted by the author. Given the priority rule assumed to guide information transmission, higher unemployment rates tend to raise the attractiveness of strong ties. Not surprisingly, as the probability of unemployment rises, personal connections in stable networks tend to involve an increasing number of strong ties and less weak links. After a certain threshold in the unemployment rate, individuals start to invest much more in strong ties than would be recommended from a group optimum perspective. This over-investment in strong ties can be interpreted as the valued-graph version of the efficiency-stability conflict ${ }^{9}$.

As mentioned in the introduction, one of the interesting insights in Granovetter [15] regards the superior performance of weak links for the flow of information within the social web when compared to stronger ties, turning those connections into the preferential highway for the diffusion of information. Such is not the whole story though, since strong links can be much more operative in the coordination of individuals as highlighted by Chwe [7] and [8]. Since strong links tend to overlap, common knowledge is facilitated and strong connections may turn out to be superior in situations that require higher-order knowledge - such as political uprisings and revolutions - whereas weak links are collectively more favorable when the phenomenon investigated requires only first-order knowledge - such as job

\footnotetext{
${ }^{8}$ Chwe [7] is not a study of strategic group formation, but it also formalizes Granovetter [15] to analyze the effect of network architecture on information transmission and coordination within a certain group.

${ }^{9}$ Valued graphs are those networks in which arcs may have different intensities
} 
acquisition information.

In the next section I set up a model to analyze the properties of a trade network formation game that incorporates the idea of strong and weak interpersonal links and shows that strong links can also be relevant to overcome limitations of "cheaper" but less reliable weak links.

\section{A Trade Network}

\subsection{The Basic Setup}

The economic situation presented in this section generalizes and extends an idea suggested in Jackson and Watts [20] and part of the analysis resembles the discussion in that paper ${ }^{10}$. The basic configuration of the economy analyzed in this section consists of a set of players $N=\{1, \ldots, n\}$ connected by the complete graph - the set of all binary subsets of $N$.

There are two goods in this economy and each agent has identical preferences represented by the Cobb-Douglas utility function $U(\mathbf{x})=x_{1} x_{2}$, where $\mathbf{x}=\left(x_{1}, x_{2}\right)$. Each individual $i$ receives a random endowment $\omega_{i} \in$ $\{(1,0),(0,1)\}$ according to a probability law that is independent and identically distributed across agents, assigning the vector $(0,1)$ with probability $p$ and, consequently, $(1,0)$ with probability $1-p$. After uncertainty is resolved, agents are allowed to trade and a Walrasian equilibrium is supposed to be established.

If there is only one agent, his or her expected utility is easily seen to be zero, as any realization yields zero utility. If there are two agents, individual expected utility is $p(1-p) / 2$, since endowments will coincide with probability $p^{2}+(1-p)^{2}$, yielding utility $1 \times 0=0$ for each agent, and will differ with probability $2 p(1-p)$, yielding the Walrasian equilibrium allocation $\{(1 / 2,1 / 2),(1 / 2,1 / 2)\}$ and utility level $(1 / 2) \times(1 / 2)=1 / 4$ for each individual. The expected utility for each member in an $n$-agent economy is shown in the appendix to be

\footnotetext{
${ }^{10}$ Jackson and Watts [20] use $\pi=1$ and $p=1 / 2$ and partially investigate stability on this specification.
} 


$$
\frac{\left[1-p^{n}-(1-p)^{n}\right]}{4} .
$$

Expected utility is higher as the number of individuals increases, since this provides greater trade opportunities. Also, utility is maximized when $p=1 / 2$ and decreases when $p$ approaches 0 or 1 . This occurs because, as $p$ approaches extreme values, population heterogeneity and thus trade opportunities tend to decrease.

In this simple economy, the trading links that bring each individual together are assumed to be perfectly reliable once endowment uncertainty is resolved and agents engage in market exchanges. As suggested earlier, one can imagine a "perfectly communicating" market like this as the limit case of a diffusion chain, in which whatever is to be diffused - in this case the trading opportunities - is certainly transmitted through the existing links. The next subsection relaxes this assumption.

\section{$3.2 \quad$ Imperfect Reliability of Links}

In this subsection, some friction is incorporated into the model by assuming that the links that bring the individuals together in the marketplace are not as reliable as we first postulated. In this case, each individual may end up trading with only part of the other agents in the economy or even not trading at all. This exercise can be justified in a variety of ways, as in real life individuals will often be confronted with search costs and transaction frictions that might prevent or significantly reduce their potential trading opportunities with other members of the community.

In order to formalize this idea, I assume that each arc in the structure postulated in the previous subsection disappears with probability $\pi$, independent from the failure or survival of other edges. For a given draw, each individual has access to the agents directly or indirectly connected to him or her and is thus assumed to trade with these agents. The market structure can then be thought of as a random graph whose realizations define each individual's trading opportunities, which will be restricted to those agents 
in his or her realized connected subgraph. The probability $\pi$ can be regarded as a measure of "reliability" for this network, as defined in Bala and Goyal [3].

A particular agent will be isolated when his or her links to the other vertices fail, which occurs with probability $\pi^{n-1}$. By a similar reasoning, this same individual will be able to trade with only one other individual with probability $(n-1) \pi^{2(n-2)}(1-\pi)$. This is because 1 . there are $n-1$ possible partners, 2 . for each of these partners, there is a probability $\pi^{2(n-2)}$ that the pair is not connected to the remaining $n-2$ vertices, and 3 . there is a $1-\pi$ probability that the two vertices form indeed a pair. I show in the appendix that a similar reasoning can be used to obtain the probability that a given vertex end up in a $k$-vertex component. Having this, an individual's expected utility in a population of size $n$ can be represented as

$$
\sum_{k=1}^{n}\left(\begin{array}{l}
n-1 \\
k-1
\end{array}\right) \phi(k, \pi) \pi^{k(n-k)} \frac{1-p^{k}-(1-p)^{k}}{4}
$$

where

$$
\phi(1, \pi)=1 \text { and } \phi(k, \pi)=1-\sum_{j=1}^{k-1}\left(\begin{array}{c}
k-1 \\
j-1
\end{array}\right) \phi(j, \pi) \pi^{j(k-j)} \text { for } k>1 .
$$

The function $\phi(k, \pi)$ represents the probability that $k$ vertices end up connected in the complete graph with probability of arc disappearance $\pi$. As can be seen by mere inspection, as $\pi \rightarrow 0$, the expression above converges to $\left[1-p^{n}-(1-p)^{n}\right] / 4$ - the expected utility when there is no noise - and, as $\pi \rightarrow 1$, the expected utility tends to zero. This agrees with the intution that, as the probability of failure approaches unity the realized network will be closer to an empty graph, with no edges, only isolated vertices. In this case, each agent will be unable to trade and will thus collect zero utility. On the other hand, the more likely it is that an edge remains intact, the closer the realized network is to the original architecture, yielding the utility level achieved in the perfect communicating market with $n$ agents. Also notice that expected utility is decreasing in $\pi$, so that more reliable environments are better for the agents involved. 
What happens if agents can somehow invest in reliable links? Can an "equilibrium" occur in which agents choose to spend resources on these ties? Moreover, does the possibility of establishing these links improve welfare? In the next subsections I try to shed some light on these questions.

\subsection{Strategic Establishment of Ties}

The trade network presented in the previous subsection introduces the possibility of unreliable connections. Links may be, in some sense, "weak". In my model, the strength of a tie is understood as a composite measure of the costs and the benefits involved. As put by Granovetter [15],

$[T]$ he strength of a tie is a combination of the amount of time, the emotional intensity, the intimacy (mutual confiding), and the reciprocal services which characterize the tie. (p.1361)

To be sure, Granovetter's definition of weak and strong ties encompasses more than simply a characterization of costs and services delivered by a link. He additionally assumes that strong ties tend to generate clusters, as two individuals that are strongly attached to a third party also tend to be connected to each other. Consequently, weak ties operate as bridges between different cliques and tend to traverse society faster. In this model, I characterize the strength of a tie according to its reliability and costs. Though Granovetter's notion of strength is broader than the simple balance of costs and benefits, this remains as a close characterization to his more including definition since, whatever it is that must be transmitted, it will more certainly be diffused through strong links, though probably to a much smaller number of people than when weak ties transport it. My characterization of weak and strong ties is not distant in that sense from other formalizations of Granovetter's suggestion, as is the case of Boorman [5], in which a priority rule that favors strong connections governs the transmission of job information. In his model, the strength can also be measured according to the reliability (probability of transmission) and costs of a link.

In a trade setting, agents invest in link strength by engaging in activities to attenuate the type of frictions that cause "communication" to be occa- 
sionally unreliable. Such would be, for instance, the case of frequent trips to the marketplace to check potential opportunities or constant updating of commercial connections. People spend time "networking". In a similar framework, one can imagine the formation of insurance networks in developing economies in order to overcome frictions that would be unavoidable otherwise in these societies. This is the case, for example, of the phenomenon investigated by Fafchamps and Lund [13] in rural Philippines - a setting in which formal markets for insurance are very rare (one could loosely interpret this as a high $\pi$ in my model) and in which informal insurance networks (strong ties) tend to guarantee a relatively smooth consumption profile.

In my model, weak links are represented by the complete graph introduced as the infrastructure of the economy in the previous subsections. Here, weak links are costless but also unreliable, because they can fail with probability $\pi$. Since costs are null, a strategic setting in which weak links are voluntary would have the complete graph as the only pairwise stable network. If any two individuals were disconnected, it would pay to form a link since this would increase the expected number of individuals directly or indirectly connected to these players in the random network. On the other hand, severing links would only deprive individuals of benefits without any savings in costs. The complete configuration is then a natural choice for the weak-link infrastructure.

On top of the weak-link network, agents are allowed to establish reliable ties (strong links) at a cost $c$ for each of the individuals involved. To better understand the strategic nuances of this assumption, let $\pi=1$ initially, so that in the absence of any voluntary establishment of ties, individuals remain isolated. After a network is established, individuals trade within components (isolated connected subgraphs). So, for a component involving players $1,2,3$, structures $\{\{1,2\},\{2,3\}\}$ and $\{\{1,2\},\{2,3\},\{1,3\}\}$ yield the same trading possibilities although in the second configuration 1 and 2 incur in excessive link costs.

Using the jargon previously introduced in this paper, the object under consideration is a strategic network formation game $\Gamma=\left\langle N,\left(A_{i}\right)_{i \in N}, v, Y\right\rangle$, where $N=\{1, \ldots, n\}$ and $Y_{i}=\mathrm{EU}(G)-c \mu_{i}(G)$ denotes the expected utility 
obtained under the assumption that $i$ trades only with those individuals in his or her component of the graph $G$ (denoted by $\operatorname{EU}(G)$ ) net of direct links costs $\left(\mu_{i}(G)\right.$ counts the number of direct links maintained by $i$ under network $G)$. The value function $v$ is simply $\sum_{i=1}^{n} \mathrm{EU}(\mathbf{x}, G)-c \mu_{i}(G)$. Each individual's strategy space $A_{i}$ lists the possible links that he or she may establish with the other players of the game. In this subsection I allow players to establish ties to any other element.

I focus on the concept of pairwise stability, introduced by Jackson and Wolinsky [21]. The following proposition characterizes the stability of this network game.

Proposition 1 In the trade network game $\Gamma=\left\langle N,\left(A_{i}\right)_{i \in N}, v, Y\right\rangle$ :

(i) For $c>\frac{p(1-p)}{2}$, the unique pairwise stable network is the empty network.

(ii) For $c \leq \frac{p(1-p)}{2}$, let $k^{*}$ be such that

$$
\frac{p^{k^{*}}(1-p)+(1-p)^{k^{*}} p}{4}<c \leq \frac{p^{k^{*}-1}(1-p)+(1-p)^{k^{*}-1} p}{4} .
$$

- If $k^{*} \geq n$, any architecture connecting all vertices through $n-1$ edges is a pairwise stable configuration ${ }^{11}$.

- If $k^{*}<n$ and $\left\{p^{k^{*}}\left(1-p^{\min \left\{k^{*}, n-k^{*}\right\}}\right)+(1-p)^{k^{*}}\left[1-(1-p)^{\min \left\{k^{*}, n-k^{*}\right\}}\right]\right\} / 4 \leq$ $c$, then the architecture with int $\left[\frac{n}{k^{*}}\right]$ components with $k^{*}$ vertices and $k^{*}-1$ edges and one component with the remaining $n-k^{*} \operatorname{int}\left[\frac{n}{k^{*}}\right]$ vertices and $n-k^{*} \operatorname{int}\left[\frac{n}{k^{*}}\right]-1$ edges is a pairwise stable architecture.

- If $k^{*}<n$ and $\left\{p^{k^{*}}\left(1-p^{\min \left\{k^{*}, n-k^{*}\right\}}\right)+(1-p)^{k^{*}}\left[1-(1-p)^{\min \left\{k^{*}, n-k^{*}\right\}}\right]\right\} / 4>$ $c$, then there is no pairwise stable architecture.

Proof. See Appendix.

In the proposition, int [.] stands for the largest integer that is smaller than the argument. The integer $k^{*}$ is the critical size of a network above which an

\footnotetext{
${ }^{11}$ Architectures with $n$ vertices and $n-1$ edges comprise the lines, the stars and the half-stars, of which $\{12,23,34,45\} ;\{12,13,14,15\}$ and $\{12,13,14,45\}$, respectively, are illustrations for the case in which $n=5$ (ij is shorthand notation for $\{i, j\}$ ).
} 
extra member will yield a net negative contribution to the individual that contemplates establishing a connection with him or her. It is well-defined and unique as discussed in the proof for Proposition 1. The intuition for the result is the following:

1. In a pairwise stable graph, components with $k$ vertices are linked by at most $k-1$ vertices. If this is not the case, at least one of the members could dissolve ties and still trade with the rest of the elements in his or her component.

2. When linkage costs are too large (case i), no pairwise incentives exist for the formation of a partnership and, if any exists, members have incentives to sever links with any element in the extremes since their marginal contribution can be seen to be no larger than $\frac{p(1-p)}{2}$. In this case the only architecture that survives the pairwise stability criteria is the empty graph $\left(G^{N}=\emptyset\right)$, in which there are no edges.

3. If linkage costs are lower (case ii) than $p(1-p) / 2$, the empty graph is not pairwise stable since there are net gains in forming at least a pair $(p(1-p) / 2-c>0)$. Individuals are added to the group as long as the marginal benefit brought by each new member is not inferior to the connection costs. Having as many groups as possible satisfying this marginal criterion, there are basically two possibilities. One is that putting together two of these groups using one vertex in each team is individually profitable for the agents establishing the connection. In this case, no pairwise stable network exists, since there will be incentives to eliminate the individuals in the extremes of the larger component created. Since in the smaller separate components there would be no marginal incentives to add new elements, in the large coalition individuals with only one link are certain to yield a negative net benefit to those that are connecting them. The other possibility is that forming the large group is not pairwise incentive compatible, in which case the configuration is pairwise stable.

The table below examplifies the pairwise stability issue for all ranges of $c$ and 
for $n=3$ and $n=4$ (whose elements are generically represented by $i, j, k$ and $i, j, k, l$, respectively). For the representation of the network structures, I group elements together to represent a component and separate components by ' + ' (so $i j+k$ is the graph $\{\{i, j\}\}$ on $\{i, j, k\}$, i.e., one pair and one isolated vertex). Notice that the "connectedness" of society increases as $c$ decrease. This fact can also be inferred from Proposition 1 , since $k^{*}$ increases as $c$ gets smaller.

Table 1: Pairwise Stable Networks

\begin{tabular}{c|c|c}
\hline Linkage Costs $(c)$ & $\mathbf{n}=\mathbf{3}$ & $\mathbf{n}=\mathbf{4}$ \\
\hline$\left(\frac{p(1-p)}{2}, \infty\right)$ & $\mathrm{i}+\mathrm{j}+\mathrm{k}$ & $\mathrm{i}+\mathrm{j}+\mathrm{k}+\mathrm{l}$ \\
\hline$\left[\frac{p(1-p)[1-p(1-p)]}{2}, \frac{p(1-p)}{2}\right]$ & $i j+k$ & $i j+k l$ \\
\hline$\frac{\left(\frac{p(1-p)}{4}, \frac{p(1-p)[1-p(1-p)]}{2}\right)}{4}$ & $i j+k$ & No Stable Graph \\
\hline$\left[\frac{1-p^{4}-(1-p)^{4}-3 p(1-p)}{4}, \frac{p(1-p)}{4}\right]$ & $\mathrm{ijk}$ & $\mathrm{ijk}+\mathrm{l}$ \\
\hline$\left[0, \frac{1-p^{4}-(1-p)^{4}-3 p(1-p)}{4}\right]$ & $\mathrm{ijk}$ & ijkl \\
\hline
\end{tabular}

Two questions arise in this setting. Are stable networks efficient? Also, are efficient graphs stable? The answer to both questions is negative, which illustrates the tension between efficiency and stability that is so pervasive in network formation games.

Firstly, to investigate the possibility of inefficient stable arrangements, consider the case of $n=4$. Suppose also that $\frac{p(1-p)[1-p(1-p)]}{2}<c<\frac{p(1-p)}{2}$, so that two separate pairs of connected individuals form a pairwise stable network (see Proposition 1). Notice that

$$
\begin{array}{r}
c<\frac{p(1-p)}{2} \Rightarrow c<p(1-p)[1-p(1-p)] \Leftrightarrow \\
\Leftrightarrow 2 c<(1-p)\left(2 p-2 p^{2}+2 p^{3}\right) \Leftrightarrow \\
\Leftrightarrow 2 c<(1-p)\left[\left(1+p^{2}+p+p^{3}\right)-2 p-\left(1-3 p+3 p^{2}-p^{3}\right)\right] \Leftrightarrow \\
\Leftrightarrow 2 c<\left[(1-p)(1+p)\left(1+p^{2}\right)-2 p(1-p)-(1-p)^{4}\right] \Leftrightarrow \\
\Leftrightarrow 2 p(1-p)-4 c<1-p^{4}-(1-p)^{4}-6 c \Leftrightarrow \\
\Leftrightarrow 2\left\{2\left[\frac{1-p^{2}-(1-p)^{2}}{4}\right]-2 c\right\}<\left\{4\left[\frac{1-p^{4}-(1-p)^{4}}{4}\right]-6 c\right\}
\end{array}
$$

The left hand side in the last inequality can be seen to be the value $v$ of the stable architecture - two separate pairs - while the right hand side depicts 
the value $v$ of a line connecting the four vertices. Since the latter is larger, the stable two-pair architecture cannot be strongly efficient.

In this particular case, the two-pair architecture can be Pareto efficient. But pairwise stable architectures can fail to satisfy this efficiency requirement as well when costs are sufficiently low. Consider, for instance, linkage costs to be $c=\frac{p(1-p)[1-p(1-p)]}{2}+\epsilon$, where $0<\epsilon<\min \left\{\frac{1}{64}, \frac{p^{2}(1-p)^{2}}{2}\right\}$. This guarantees that $c$ is still within the range where separate pairs are pairwise stable networks. Under this assumption the individual net benefit of a shift from the pairwise stable configuration to a graph that connects all the agents in the economy through a line is at least

$$
\begin{gathered}
\frac{1-p^{n}-(1-p)^{n}}{4}-\frac{1-p^{2}-(1-p)^{2}}{4}-c=\frac{1-p^{n}-(1-p)^{n}}{4}-\frac{p(1-p)}{2}-\frac{p(1-p)[1-p(1-p)]}{2}-\epsilon> \\
>\frac{1-p^{n}-(1-p)^{n}}{4}-\frac{p(1-p)}{2}[2-p(1-p)]-\frac{1}{64} \geq \frac{1-p^{n}-(1-p)^{n}}{4}-\frac{15}{64} .
\end{gathered}
$$

Since $15 / 64<16 / 64=1 / 4=\lim _{n \rightarrow \infty}\left[1-p^{n}-(1-p)^{n}\right] / 4$, the above expression is positive for sufficiently large $n$, so that all individuals can be made better off.

As for the second question, it is also easy to see that efficient networks might not be stable. If $\frac{p(1-p)}{4}<c<\frac{p(1-p)[1-p(1-p)]}{2}$, for instance, Proposition 1 asserts that there are no stable networks if $n \geq 4$. But a Pareto efficient network always exists since there is a finite number of vertices and thus a finite set of possible graphs (which are the subsets of the complete graph $G^{N}$ ) on which Pareto dominance corresponds to a transitive and asymmetric partial ordering. A Pareto efficient finite graph does not exist if and only if the Pareto dominace relation is cyclic and complete. But if this is so, the dominance relation fails to be transitive and asymmetric. Then, when $\frac{p(1-p)}{4}<c<\frac{p(1-p)[1-p(1-p)]}{2}$, there is a Pareto efficient network that fails to be pairwise stable. Likewise, there may be strongly efficient networks that fail to be pairwise stable. Since the set of possible graphs is finite, the value function will attain a maximum for any given value of $c$, so there always exists a strongly efficient network. It fails to be stable in the range of costs where no graph is pairwise stable. But even in the case when there is a stable configuration the strongly efficient graph may not be an equilibrium, as can be seen from the example of $n=4$ presented earlier when 
$\frac{p(1-p)[1-p(1-p)]}{2}<c<\frac{p(1-p)}{2}$.

In the next subsection I drop the assumption that $\pi=1$ and investigate the behavior of strong ties in the presence of imperfectly reliable links.

\subsection{The Strength of Strong Ties}

In this section I drop the assumption that $\pi=1$ and investigate two questions. Can we still expect strong links to be formed in equilibrium once we allow for the occurrence of unreliable connections? And, in case agents choose to invest in ties, does the equilibrium improve upon the situation in which no strong links are allowed?

The strategic establishment of reliable links under the presence of unreliable connections $(0<\pi<1)$ parallels, in all aspects, the investigation carried out in the last subsection (where $\pi=1$ ). As a matter of fact, the following proposition, which is very similar in nature to Proposition 1, characterizes pairwise stability for this strategically restricted case. For the sake of clarity I increase the description of the strategic situation at hand by adding $\pi$ to the original tuple: $\Gamma=\left\langle N,\left(A_{i}\right)_{i \in N}, v, Y, \pi\right\rangle$.

In order to state the Proposition, let

$$
E U(k, \pi)=\sum_{j=0}^{n-k}\left(\begin{array}{c}
n-k \\
j
\end{array}\right) \pi^{(k+j)(n-k-j)} \Theta(k, j, \pi) \frac{1-p^{k+j}-(1-p)^{k+j}}{4}
$$

where

$$
\begin{aligned}
& \Theta(k, 0, \pi)=1, \\
& \Theta(k, 1, \pi)=1-\pi^{k} \text { and, for } j>1, \\
& \Theta(k, j, \pi)=1-\sum_{i=1}^{j}\left(\begin{array}{c}
j-1 \\
i-1
\end{array}\right) \phi(i, \pi) \pi^{i(k+j-i)}-\sum_{i=1}^{j-1} \Theta(k, i, \pi) \pi^{(j-i)(k+i)}
\end{aligned}
$$

with $\phi(i, \pi)$ defined as in subsection 3.2. The function $\Theta(k, j, \pi)$ is the probability that a $k$-vertex component and $j$ isolated vertices end up connected in the complete graph where the probability of arc disappearance is $\pi$. It can be seen that, when $k=1, \Theta(1, j, \pi)=\phi(1+j, \pi)$. The proposition now is stated as: 
Proposition 2 In the trade network game $\Gamma=\left\langle N,\left(A_{i}\right)_{i \in N}, v, Y, \pi\right\rangle$ :

(i) For $c>E U(2, \pi)$, the only pairwise stable network is the empty network.

(ii) For $c \leq E U(2, \pi)$,

- If $E U(n, \pi)-E U(n-1, \pi) \geq c$, any architecture connecting all vertices through $n-1$ edges is a pairwise stable configuration.

- If $E U(n, \pi)-E U(n-1, \pi)<c$, define $k^{*}$ such that

$$
E U\left(k^{*}+1, \pi\right)-E U\left(k^{*}, \pi\right)<c \leq E U\left(k^{*}, \pi\right)-E U\left(k^{*}-1, \pi\right)
$$

- If $n / 2<k^{*}<n$ and $E U(n, \pi)-E U\left(k^{*}, \pi\right)<c$, then the architecture of one component with $k^{*}$ vertices and $k^{*}-1$ edges and one component with the remaining $n-k^{*}$ vertices and $n-k^{*}-1$ edges is a pairwise stable architecture. If the inequality is reversed to $>$, then there is no pairwise stable network.

- If $k^{*} \leq n / 2$ and $E U\left(2 k^{*}, \pi\right)-E U\left(k^{*}, \pi\right)<c$, then the architecture with int $\left[\frac{n}{k^{*}}\right]$ components with $k^{*}$ vertices and $k^{*}-1$ edges and one component with the remaining $n-k^{*}$ int $\left[\frac{n}{k^{*}}\right]$ vertices and $n-k^{*} \operatorname{int}\left[\frac{n}{k^{*}}\right]-$ 1 edges is a pairwise stable architecture. If the inequality is reverted to $\dot{\&}$, then there is no pairwise stable network.

Proof. See Appendix.

The above proposition reduces to Proposition 1 if $\pi=1$ and this allows us to look at this new game in a very similar manner. Notice that, as individuals can count on the possibility of link formation with an imperfect though positive degree of confidence, the existence of (costless) weak ties poses additional disincentives for the connection of two given players. As a matter of fact, as $\pi \rightarrow 0$ the (strong-link) empty graph, in which nobody voluntarily establishes links with the others, becomes pairwise stable at 
lower and lower costs. It can actually be pointed out that the thresholds in Proposition 2 are smaller than those in Proposition 1 for any positive $\pi$ : the "connectedness" of society (through strong links) is lower.

The first question can then be answered affirmatively: the incorporation of weak links at no (or at very low) costs does not hinder the establishment of strong ties between the agents of the economy. It rather introduces a new source of disincentive for the formation of such connections on top of the direct cost of linkage. In this sense, the noisier (high $\pi$ ) the weak tie structure and/or the less costly it is to create strong ties (low $c$ ) under a particular scenario, the more likely it is that any network will voluntarily emerge as an equilibrium - i.e. a pairwise stable configuration.

The answer for the second question in this subsection is positive: agents are made better off in equilibrium when they are allowed to invest in contacts. As a matter of fact, every individual is at least as well off as in the empty graph. This follows directly from the definition of pairwise stability. If the empty graph is not pairwise stable and there is a non-empty graph that is pairwise stable, the establishment of links must be at least neutral to both parties. This implies that a link will be established only when it yields non-negative net benefits to both vertices. On the other hand, if a link provides negative benefits to at least one of the vertices involved, there will be incentives to sever this connection. Every individual must then collect non-negative utility from a pairwise stable network. This is a straightforward result, which nevertheless deserves to be mentioned since not rarely equilibrium configurations are suboptimal when the ideal conditions that guarantee the First Welfare Theorem do not apply, which is the case here.

\section{Conclusion}

In this paper, I use results from the literature on strategic formation of networks and use it to study a particular economic situation. A number of social institutions are often represented by a web of interpersonal links that brings agents together and coordinates some collective outcome. Following this insight, the harmonization of trade opportunities within a given 
population is here represented by a structure of interagent ties that brings individuals together in the marketplace for the exchange of goods. The possibility of search and transaction frictions may nevertheless attenuate the cohesion of such a networking structure and cause market opportunities not to be entirely explored.

Under such circumstances it is not implausible that society will seek alternative mechanisms to overcome such communication failures. I speculate on the possibilities of voluntary networking efforts by the agents of a given community for the formation of costly though more reliable connections with other members of society in order to seize any trade opportunity more effectively. As a matter of fact, some evidence exists that such a reaction is not uncommon in underdeveloped societies (e.g. Fafchamps and Lund [13] and references therein) or small specialized markets, where formal market institutions are often substantially noisy.

Using a simple model that extends and generalizes an example suggested in Jackson and Watts [21], I find that in fact such voluntary networks of "strong ties" can usually be sustained as a stable outcome, though examples are not hard to achieve in which no equilibrium configuration occurs. Nevertheless, whenever such a structure exists it improves general well-being over a situation in which only formal unreliable markets existed.

Though voluntary networking efforts are no substitute for an improvement in the reliability of formal institutions $(\pi \rightarrow 0)$, the analysis indicates that the emergence of informal insurance networks or extensive investment in connections should come as no surprise in the presence of "noisy" market institutions. 


\section{Appendix}

\section{Expected utility for an $n$-member market}

For each endowment realization, a Walrasian equilibrium consists of a priceallocation pair $\left\langle\mathbf{p}=\left(p_{1}, p_{2}\right),\left\{\mathbf{x}=\left(x_{1}^{i}, x_{2}^{i}\right)\right\}_{i=1}^{n}\right\rangle$ for which 1. preferences are maximized and 2. markets clear. More formally,

1. $\forall i, \mathbf{x}_{i}=\operatorname{argmax}_{\mathbf{y}} U(\mathbf{y})$ s.t. $\mathbf{p} \cdot \mathbf{y} \leq \mathbf{p} \cdot \omega_{i}$; and

2. $\sum_{i=1}^{n} \mathbf{x}_{i}=\sum_{i=1}^{n} \omega_{i}$.

For a given endowment realization there are basically two types of agents in this economy: those with endowment $(1,0)$ and those with endowment $(0,1)$. Let $n_{1}$ and $n_{2}$ be the number of individuals with endowment vectors $(1,0)$ and $(0,1)$, respectively $\left(n_{1}+n_{2}=n\right)$. Let $\overline{\mathbf{x}}=\left(\bar{x}_{1}, \bar{x}_{2}\right)$ denote the consumption of $(1,0)$-individuals and $\underline{\mathrm{x}}=\left(\underline{x}_{1}, \underline{x}_{2}\right)$ represent the bundle consumed by $(0,1)$-individuals. The two conditions above can then be translated into:

$$
\begin{array}{rc}
p_{1} x_{1}^{i}=p_{2} x_{2}^{i}, \forall i & \text { (preference maximization) } \\
p_{1}=\mathbf{p} \cdot \overline{\mathbf{x}} & \text { (preference maximization) } \\
p_{2}=\mathbf{p} \cdot \underline{\mathbf{x}} & \text { (preference maximization) } \\
n_{1} \bar{x}_{1}+n_{2} \underline{x}_{1}=n_{1} & \text { (market clearing) } \\
n_{1} \bar{x}_{2}+n_{2} \underline{x}_{2}=n_{2} & \text { (market clearing) }
\end{array}
$$

We concentrate on the analysis of the Walrasian equilibrium allocations for each individual. From (1) and (2) one gets $\bar{x}_{1}=1 / 2$. Multiplying (4) by $p_{1}$ and using $\bar{x}_{1}=1 / 2$ we arrive at $\underline{x}_{1}=n_{1} / 2 n_{2}$. Proceeding analogously, $\underline{x}_{2}=1 / 2$ and $\bar{x}_{2}=n_{2} / 2 n_{1}$, so that $\overline{\mathbf{x}}=\left(1 / 2, n_{2} / 2 n_{1}\right)$ and $\underline{\mathbf{x}}=\left(n_{1} / 2 n_{2}, 1 / 2\right)$. Agents with endowment $(1,0)$ then get utility $n_{2} / 4 n_{1}$, whereas agents with endowment $(0,1)$ get utility $n_{1} / 4 n_{2}$. Averaging over all the possibilities, the expected utility of any given individual in an $n$-agent economy given that his of her endowment is $(0,1)$ is expressed by 


$$
\sum_{k=1}^{n-1}\left(\begin{array}{c}
n-1 \\
k
\end{array}\right) p^{n-k-1}(1-p)^{k} \frac{k}{4(n-k)},
$$

whereas his or her expected utility given a $(1,0)$ endowment is

$$
\sum_{k=1}^{n-1}\left(\begin{array}{c}
n-1 \\
k
\end{array}\right) p^{k}(1-p)^{n-k-1} \frac{k}{4(n-k)}
$$

The (unconditional) expected utility is then $p \times(6)+(1-p) \times(7)$. Before putting these two pieces together - and in order to obtain a simple representation for this expression-notice that:

$$
\begin{array}{r}
(1-p) \times \sum_{k=1}^{n-1}\left(\begin{array}{c}
n-1 \\
k
\end{array}\right) p^{k}(1-p)^{n-k-1} \frac{k}{4(n-k)}= \\
=\sum_{k=1}^{n-1}\left(\begin{array}{c}
n-1 \\
k
\end{array}\right) p^{k}(1-p)^{n-k} \frac{k}{4(n-k)}= \\
=\sum_{k=1}^{n-1}\left(\begin{array}{c}
n-1 \\
n-k-1
\end{array}\right) p^{k}(1-p)^{n-k} \frac{k}{4(n-k)}= \\
=\sum_{k=1}^{n-1} \frac{(n-1) !}{(n-k-1) ! k !} p^{k}(1-p)^{n-k} \frac{k}{4(n-k)}= \\
=\sum_{k=1}^{n-1} \frac{(n-1) ! \times k \times(n-k)}{(n-k-1) ! k ! \times(n-k) \times k} p^{k}(1-p)^{n-k} \frac{k}{4(n-k)}= \\
=\sum_{k=1}^{n-1} \frac{(n-1) !}{(n-k) !(k-1) !} \times \frac{(n-k)}{k} p^{k}(1-p)^{n-k} \frac{k}{4(n-k)}= \\
=\sum_{k=1}^{n-1}\left(\begin{array}{c}
n-1 \\
n-k
\end{array}\right) p^{k}(1-p)^{n-k} \frac{(n-k)}{k} \times \frac{k}{4(n-k)}= \\
=\sum_{k=1}^{n-1}\left(\begin{array}{l}
n-1 \\
n-k
\end{array}\right) p^{k}(1-p)^{n-k} \frac{1}{4}= \\
=\sum_{j=1}^{n-1}\left(\begin{array}{c}
n-1 \\
j
\end{array}\right) p^{n-j}(1-p)^{j} \frac{1}{4} .
\end{array}
$$

The expression we are interested in then reduces to: 


$$
\begin{array}{r}
\sum_{k=1}^{n-1}\left(\begin{array}{c}
n-1 \\
k
\end{array}\right) p^{n-k}(1-p)^{k}\left(\frac{k}{4(n-k)}+\frac{1}{4}\right)= \\
=\sum_{k=1}^{n-1}\left(\begin{array}{c}
n-1 \\
k
\end{array}\right) p^{n-k}(1-p)^{k} \frac{n}{4(n-k)}= \\
=\frac{1}{4} \sum_{k=1}^{n-1}\left(\begin{array}{l}
n \\
k
\end{array}\right) p^{n-k}(1-p)^{k}= \\
=\frac{1}{4}\left[\sum_{k=0}^{n}\left(\begin{array}{l}
n \\
k
\end{array}\right) p^{n-k}(1-p)^{k}-p^{n}-(1-p)^{n}\right]= \\
=\frac{\left[1-p^{n}-(1-p)^{n}\right]}{4},
\end{array}
$$

as claimed.

\section{Expected utility for an unreliable complete $n$-network}

Consider a set of $k$ vertices and fix one of the elements in the set. The events that correspond to the occurrence of more than one connected component can be framed in terms of the fixed element as follows:

- If the element is isolated from the other vertices;

- If the element forms a two-vertex component isolated from the other vertices;

- If the element forms a three-vertex component isolated from the other vertices;

- ...

- If the element forms a $k$-1-vertex component isolated from the remaining vertex.

If the probability that the $k$ vertices end up connected is denoted by $\phi(k, \pi)$, it should be one minus the probability that the above events happen. This means that 


$$
\begin{aligned}
\phi(k, \pi) & =1-\left[\pi^{k-1}+\left(\begin{array}{c}
k-1 \\
1
\end{array}\right) \phi(2, \pi) \pi^{2(k-2)}+\left(\begin{array}{c}
k-1 \\
2
\end{array}\right) \phi(3, \pi) \pi^{3(k-3)}+\ldots\right]= \\
& =1-\sum_{j=1}^{k-1}\left(\begin{array}{c}
k-1 \\
j-1
\end{array}\right) \phi(j, \pi) \pi^{j(k-j)}
\end{aligned}
$$

where $\phi(1, \pi)=1$. The sum between brackets in the first line represents the probability that the $k$ vertices are not connected. This happens when the fixed element is isolated (with probability $\pi^{k-1}$ ); or when it forms a pair with a given element (with probability $\phi(2, \pi)$ and for $\left(\begin{array}{c}k-1 \\ 1\end{array}\right)$ potential partners) and this pair is isolated from the remaining $k-2$ vertices (with probability $\left.\pi^{2(k-2)}\right)$; or when it forms a triple with two given elements... Notice that $\phi(k, \pi) \rightarrow 1, \forall k$ as $\pi \rightarrow 0$ and that $\phi(k, \pi) \rightarrow 0, \forall k \geq 2$ as $\pi \rightarrow 1$.

An individual will end up in a $k$-vertex component when $k-1$ other vertices and he or she are isolated from the remaining $n-k$ members of the population (with probability $\pi^{k(n-k)}$ and for $\left(\begin{array}{l}n-1 \\ k-1\end{array}\right)$ potential mates) and the $k$ individuals are connected (with probability $\phi(k, \pi)$ ). The following table portrays the different trading possibilities for a given agent:

\begin{tabular}{c|c|c}
\hline $\begin{array}{c}\text { Component } \\
\text { Size }\end{array}$ & Probability & $\begin{array}{c}\text { Expected } \\
\text { Utility }\end{array}$ \\
\hline$k=1$ & $\phi(1, \pi) \pi^{n-1}$ & 0 \\
$k=2$ & $\left(\begin{array}{c}n-1 \\
1\end{array}\right) \phi(2, \pi) \pi^{2(n-2)}$ & $\frac{1-p^{2}-(1-p)^{2}}{4}$ \\
$k=3$ & $\left(\begin{array}{c}n-1 \\
2\end{array}\right) \phi(3, \pi) \pi^{3(n-3)}$ & $\frac{1-p^{3}-(1-p)^{3}}{4}$ \\
$\vdots$ & $\vdots$ & $\vdots$ \\
$k=n-1$ & $\left(\begin{array}{c}n-1 \\
n-2\end{array}\right) \phi(n-1, \pi) \pi^{n-1}$ & $\frac{1-p^{n-1}-(1-p)^{n-1}}{4}$ \\
$k=n$ & $\phi(n, \pi)$ & $\frac{1-p^{n}-(1-p)^{n}}{4}$ \\
\hline
\end{tabular}

Averaging across the different alternatives, one gets

$$
\sum_{k=1}^{n}\left(\begin{array}{l}
n-1 \\
k-1
\end{array}\right) \phi(k, \pi) \pi^{k(n-k)} \frac{1-p^{k}-(1-p)^{k}}{4}
$$

\section{Proofs and Additional Lemmas}

The following lemma will be useful in some of the propositions proved in this appendix. 
Lemma 1 Strongly (or Pareto) efficient and pairwise stable n-vertex (costlylink) network components have at most $n$-1 edges.

Proof. Suppose a (strong or Pareto) efficient network with $n$ vertices has more than $n-1$ edges. It is then possible to eliminate one of the edges while still maintaining connectedness. Since members can trade with any directly or indirectly linked player, connectedness is the only property that matters for the allocation rule $Y$. Given this, severing superfluous links will save costs for both individuals involved and will thus increase the value function (which is the sum of the individual expected utilities net of connection costs) or improve at least one member without hurting any other. But if the network is (strongly or Pareto) efficient, this is an impossibility. So, if the network is efficient, it must have at most $n-1$ edges.

For a similar reason, a network with superfluous links for purposes of trade will generate incentives for links to be severed, which would thus imply that $n$-networks with more than $n-1$ arcs will not be pairwise stable. Q.E.D.

\section{Proof of Proposition 1}

(i) According to Lemma 1, any $k$-component (connected subgraph) of a pairwise stable graph with more than two vertices has at most $k-1$ edges. Consider severing links with one of the vertices in the extremity of the component. Since $\frac{1-p^{k}-(1-p)^{k}}{4}$ is concave in $k$, this will yield a maximum cost in terms of expected utility from trade of

$$
\frac{3 p(1-p)}{4}-\frac{p(1-p)}{2}=\frac{p(1-p)}{4}
$$

which is smaller than the connection cost $c$ saved. A component of a pairwise stable network will thus have at most two vertices. If it has two vertices, the individual net benefit from the link (net of connection costs) is given by

$$
\frac{p(1-p)}{2}-c<0
$$

so this component is not pairwise stable. Consequently, if a pairwise stable architecture exists it is the empty graph. This configuration is indeed pairwise stable since, by the same cost-benefit computation above, there are no 
incentives to form links between any two individuals.

(ii) First notice that $k^{*}$ is well defined since $f(x): \aleph \rightarrow \Re$ with $x \mapsto\left\{p^{x}(1-\right.$ $\left.p)+(1-p)^{x} p\right\} / 4 \Rightarrow f(x)-f(x-1)=-\left[(1-p)^{2} p^{x-1}+p^{2}(1-p)^{x-1}\right] / 4<0 \Rightarrow f$ is decreasing. Also, $f(1)=\frac{p(1-p)}{2}$ and $\lim _{x \rightarrow \infty} f(x)=0$ from which it is easy to see that $\forall c \in\left(0, \frac{p(1-p)}{2}\right)$, there exists a unique $k^{*} \in \aleph: f\left(k^{*}\right)<c \leq$ $f\left(k^{*}-1\right)$.

The function $f$ can be rewritten as $f(k)=\frac{\left[1-p^{k+1}-(1-p)^{k+1}\right]-\left[1-p^{k}-(1-p)^{k}\right]}{4}$, which represents the expected incremental gain in a trade network with $k$ agents when an extra member is incorporated. The integer $k^{*}$ is thus the critical size of a network above which an extra member will represent a net negative contribution to the individual that contemplates establishing a connection with him or her.

Notice that $k^{*}>n \Rightarrow k^{*}-1>n-1 \Rightarrow \frac{\left[1-p^{n}-(1-p)^{n}\right]-\left[1-p^{n-1}-(1-p)^{n-1}\right]}{4}=$ $f(n-1)>f\left(k^{*}-1\right)>c$ and $\frac{\left[1-p^{k}-(1-p)^{k}\right]-\left[1-p^{k-1}-(1-p)^{k-1}\right]}{4}>c, \forall k \leq n$, so it is incentive compatible for any vertex to connect a new member to his or her component. The incentives to establish a bridge between any two disconnected components are even stronger. In consequence, any pairwise stable network should have a single component. Any architecture connecting all the vertices with $n-1$ arcs (which is a requirement of Lemma 1) is thus pairwise stable.

Starting from an empty graph, there are incentives to add new members to a group as long as the group size is smaller than $k^{*}$. Assume that the population is thus divided into as many coalitions as possible of size $k^{*}$. Within each of these groups, no member gains by severing links with other agents as long as the individuals are connected through $k^{*}-1$ edges. If the population size is not perfectly divisible by $k^{*}$, gather the remaining members in a separate group (which, having less than $k^{*}$ elements, will not engender incentives for the dissolution of links either). 
If $\frac{n}{2}<k^{*}$, two components are formed: one with $k^{*}$ members and another with $n-k^{*}$ members. When $\left\{\left[1-p^{n}-(1-p)^{n}\right]-\left[1-p^{k^{*}}-(1-p)^{k^{*}}\right]\right\} / 4=$ $\left\{p^{k^{*}}\left(1-p^{n-k^{*}}\right)+(1-p)^{k^{*}}\left[1-(1-p)^{n-k^{*}}\right]\right\} / 4<c$, no member in either group will have incentives to establish a link between the two groups. This configuration, in which each component has the internal structure dictated by Lemma 1, is thus pairwise stable.

If $k^{*} \leq \frac{n}{2}$, the organization of groups as in the previous case will generate $\operatorname{int}\left[\frac{n}{k^{*}}\right]$ components with $k^{*}$ vertices and one component with the remaining $n-k^{*} \operatorname{int}\left[\frac{n}{k^{*}}\right]$. When $\left\{\left[1-p^{2 k^{*}}-(1-p)^{2 k^{*}}\right]-\left[1-p^{k^{*}}-(1-p)^{k^{*}}\right]\right\} / 4=$ $\left\{p^{k^{*}}\left(1-p^{k^{*}}\right)+(1-p)^{k^{*}}\left[1-(1-p)^{k^{*}}\right]\right\} / 4<c$, no member of a $k^{*}$-component will have incentives to establish a connection with a vertex of another group (with $k^{*}$ vertices or less). As a consequence, this configuration is pairwise stable: no vertex will be willing to break up established ties nor to form new links.

These two cases $\left(k^{*}>n / 2\right.$ and $\left.k^{*} \leq n / 2\right)$ together deliver the result stated in the second bullet of case ii since $\left\{p^{k^{*}}\left(1-p^{\min \left\{k^{*}, n-k^{*}\right\}}\right)+(1-p)^{k^{*}}[1-(1-\right.$ $\left.\left.p)^{\min \left\{k^{*}, n-k^{*}\right\}}\right]\right\} / 4=\left\{p^{k^{*}}\left(1-p^{k^{*}}\right)+(1-p)^{k^{*}}\left[1-(1-p)^{k^{*}}\right]\right\} / 4$ if $k^{*} \leq n / 2$ and $\left\{p^{k^{*}}\left(1-p^{n-k^{*}}\right)+(1-p)^{k^{*}}\left[1-(1-p)^{n-k^{*}}\right]\right\} / 4=\left\{p^{k^{*}}\left(1-p^{k^{*}}\right)+(1-\right.$ $\left.p)^{k^{*}}\left[1-(1-p)^{k^{*}}\right]\right\} / 4$ otherwise.

For each of the cases above $\left(k^{*}>n / 2\right.$ and $\left.k^{*} \leq n / 2\right),\left\{\left[1-p^{n}-(1-p)^{n}\right]-[1-\right.$ $\left.\left.p^{k^{*}}-(1-p)^{k^{*}}\right]\right\} / 4>c$ or $\left\{\left[1-p^{2 k^{*}}-(1-p)^{2 k^{*}}\right]-\left[1-p^{k^{*}}-(1-p)^{k^{*}}\right]\right\} / 4>c$ imply that incentives will be in place for larger groups to be formed. This in turn implies that the architecture suggested will not be pairwise stable. If a pairwise stable architecture existed, it would then involve components with $k^{*}$ vertices or less (if there were more than $k^{*}$ vertices links would be severed profitably). But at the same time there are incentives for any group with $k^{*}$ members or less to merge with other components. By the definition of $k^{*}$ the expected benefit from the incorporation of a single vertex would be superior to the linkage cost $c$ and the establishment of a bridge with another component with two or more vertices would be even more so. So, there is 
no pairwise stable architecture. Q.E.D.

The following Lemma is used in proving Proposition 2.

Lemma 2 For fixed $\pi$ and $n, f(k)=E U(k, \pi)$ is a concave function of $k$.

Proof. Given $n$ and $\pi,(E U(k, \pi))_{k=1}^{n}$ can be seen as a point in $\Re^{n}$, the $n$-dimensional Euclidian space. Furthermore, $\pi(\in[0,1]) \mapsto \mathbf{f}(\pi)=$ $(E U(k, \pi))_{k=1}^{n}\left(\in \Re^{n}\right)$ is a continuous function (each component is basically a polynomial in $\pi)$.

Let $S=\left\{\mathbf{x}=\left(x_{1}, \ldots, x_{n}\right) \in \Re^{n} \mid x_{k+1}-x_{k} \leq x_{k}-x_{k-1}, k=2, \ldots, n-1\right\}$, so that $S$ is the (closed) set of all (weakly) concave functions with domain $\{1, \ldots, n\}$ (which are thus representable as vectors). Notice that $E U(k, 1)=$ $\frac{1-p^{k}-(1-p)^{k}}{4}$ is a strictly concave function with domain $\{1, \ldots, n\}$ (as long as $p \in(0,1)$ and thus belongs to the interior of $S$ (denoted $S \backslash \partial S$ ). On the other hand, $E U(k, 0)=\frac{1-p^{n}-(1-p)^{n}}{4}, \forall k$, which is a constant function with domain $\{1, \ldots, n\}$ and thus belongs to the boundary of $S$ (denoted $\partial S$ ).

Using Theorem 4.22 in Rudin [27] (p.93), $\mathbf{f}([0,1])$ is a connected subset of $\Re^{n}$. If $\mathbf{f}([0,1]) \subset S$, nothing remains to be shown. If otherwise $\mathbf{f}([0,1])$ is not a subset of $S$, since $\mathbf{f}(1) \in S \backslash \partial S, \mathbf{f}(0) \in \partial S$ and $\mathbf{f}([0,1])$ is connected, $\exists \pi^{*} \in(0,1)$ such that $\mathbf{f}\left(\pi^{*}\right) \in \partial S$. This means that $\exists k \in\{2, \ldots, n-1\}$ such that $E U\left(k+1, \pi^{*}\right)-E U\left(k, \pi^{*}\right)=E U\left(k, \pi^{*}\right)-E U\left(k-1, \pi^{*}\right)$. But this can be seen to be impossible for any $\pi \in(0,1)$. Q.E.D.

\section{Proof of Proposition 2}

The proof parallels that of Proposition 1 and therefore is omitted. It is easily reproduced once one realizes that, given a population of size $n$,

1. $\Theta(k, j, \pi)$ is the probability that a $k$-member strong-link network and $j$ isolated vertices end up connected in the complete graph with probability of arc disappearance $\pi$;

2. $E U(k, \pi)$ is the expected utility from trade for a strong-link network with $k$ vertices; and

3. $E U(k, \pi)$ is a concave function of $k$ (Lemma 2). 
The derivation of $\Theta(k, j, \pi)$ parallels that of $\phi(k, \pi)$. It basically computes the probability that $k$-member strong-link network and $j$ isolated vertices end up connected by fixing one of the isolated points and framing the complemetary events in terms of this vertex. The $k+j$ vertices would end up disconnected

- If the fixed element is isolated from the other $k+j-1$ vertices;

- If the fixed element forms a two-vertex component with another of the isolated $j$ vertices and this pair is isolated from the other $k+j-2$ vertices;

- If the fixed element forms a link with the $k$-component and is isolated from the other $j-1$ vertices;

- If the element forms a three-vertex component with other two of the $j$ isolated vertices and is isolated from the other $k+j-3$ vertices;

- If the fixed element forms a component with the $k$-component and another of the $j$ isolated vertices and is isolated from the other $j-2$ vertices;

- etc.

Using $\Theta(k, j, \pi)$, the computation of the expected utility from trade when the individual is the member of a $k$-vertex strong-link component is easily seen to be $E U(k)$. The concavity of this function allows the utilization of exactly the same rationale adopted in the proof of Proposition 1 to prove this proposition. 


\section{References}

[1] Akerlof, G. (1997), "Social Distance and Social Decisions", Econometrica, 65, 5, 1005-1027.

[2] Bala, V. and S.Goyal (2000), "Self-Organization in Communication Networks", Econometrica, 68, 1181-1230.

[3] Bala, V. and S.Goyal (2000), "A Strategic Analysis of Network Reliability", Review of Economic Design, 5, 205-228.

[4] Bernheim, B.D., B.Peleg and M.Whinston (1987) "Coalitionproof Nash Equilibria I. Concepts.", Journal of Economic Theory, 42, $1-12$.

[5] Boorman, S.A. (1975), "A Combinatorial Optimization Model for Transmission of Job Information through Contact Networks", Bell Journal of Economics, 6, 1, 216-249.

[6] Buchanan, J. (1965), "An Economic Theory of Clubs", Economica, 32, 125, 1-14.

[7] Chwe, M. (2000) "Communication and Coordination and Social Networks", Review of Economic Studies, 62, 1-16.

[8] Chwe, M. (2001) Rational Ritual: Culture, Coordination and Common Knowledge, Princeton: Princeton University Press.

[9] Corominas-Bosch (1999) "On Two-Sided Network Markets", Ph.D. Dissertation: Universitat Pompeu Fabra.

[10] Dutta, B. and M.Jackson (2000) "The Stability and Efficiency of Directed Communication Networks", Review of Economic Design, 5, 251-272.

[11] Dutta, B. and M.Jackson (eds.), forthcoming, Models of the Strategic Formation of Networks and Graphs, Heidelberg: Springer-Verlag. 
[12] Dutta, B. and S. Mutuswami (1997), "Stable Networks", Journal of Economic Theory, 76, 322- 344.

[13] Fafchamps, M. and S. Lund (2003), "Risk-Sharing Networks in Rural Philippines," Journal of Development Economics, 71(1), 261-287.

[14] Genicot, G. and D. Ray (2003) "Endogenous Group Formation in Risk-Sharing Arrangements", Review of Economic Studies, 70, 87-113.

[15] Granovetter, M. (1973), "The Strength of Weak Ties", American Journal of Sociology, 78, 1360-1380.

[16] Granovetter, M. (1995), Getting a Job: A Study of Contacts and Careers, Second Edition, Chicago: University of Chicago Press.

[17] Haller, H. and S.Sarangi (2005), "Nash Networks with Heterogeneous Links", Mathematical Social Sciences, 50(2), 181-201.

[18] Jackson, M. (2001), "The Stability and Efficiency of Economic and Social Networks", forthcoming in Sertel, M. (ed.) Advances in Economic Design, Heidelberg: Springer-Verlag.

[19] Jackson, M. (2010), Social and Economic Networks, Princeton University Press.

[20] Jackson, M. and A. Watts (2002), "The Evolution of Social and Economic Networks," Journal of Economic Theory, 106(2), 265-295.

[21] Jackson, M. and A. Wolinsky (1996), "A Strategic Model of Social and Economic Networks", Journal of Economic Theory, 71, 44-74.

[22] Johnson, C. and R.P.Gilles (2000), "Spacial Social Networks", Review of Economic Design, 5, 273-300.

[23] Kranton, R. and D. Minehart (2000), "A Theory of Buyer-Seller Networks" Review of Economic Design, 5, 301-331.

[24] Myerson, R. (1977), "Graphs and Cooperation in Games", Mathematics of Operations Research, 2, 225-229. 
[25] Postlewaite, A. (2001), "Social Arrangements and Economic Behavior", Annals of Economics and Statistics, No.63-64, 67-87.

[26] Rogers, E. and F. Shoemaker (1971), The Communication of Innovations, Second Edition, New York: Free Press.

[27] Rudin, W. (1976), Principles of Mathematical Analysis, Third Edition, New York: McGraw-Hill Book Company.

[28] Tiebout, C. (1956), "A Pure Theory of Local Expenditures", Journal of Political Economy, 64, 5, 416-424.

[29] Topa, G. (2001), " Social Interactions, Local Spillovers and Unemployment", Review of Economic Studies, 68, 2, 261-295.

[30] Wasserman, S. and K. Faust (1994), Social Network Analysis: Methods and Applications, Cambridge: Cambridge University Press. 


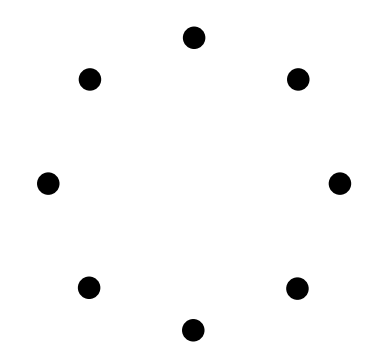

Figure 1A

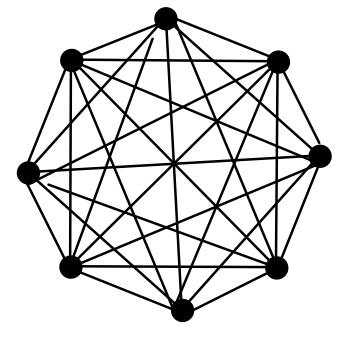

Figure 1B

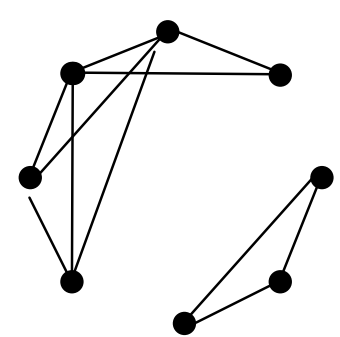

Figure 1C 